\title{
Die Geschichte des Hydrobiologischen Laboratoriums in Kastanienbaum
}

\author{
Von Hein Rich WolfF, Luzern \\ Manuskript eingegangen am 29. Dezember 1965
}

Angeregt durch die Erforschung des Weltmeers und die immer häufiger auftretenden Studien an Binnengewässern des Auslandes, haben schweizerische Naturwissenschaftler in den drei letzten Jahrzehnten des vergangenen Jahrhunderts Untersuchungen an Schweizer Seen durchgeführt.

Es erschienen Publikationen von ASPER 1881-1889, ImhoF 1883-1893, HeusChe R 1890-1895 und F. A. FOREL 1876-1904, insbesondere sein dreibändiges Werk Le Léman, 1882-1904. Hauptsächlich durch die Arbeiten Forels wurden Mitglieder der Naturforschenden Gesellschaft Luzern angeregt, ihr Interesse dem Vierwaldstättersee zuzuwenden. Apotheker SuIDTER hielt 1883-1886 Vorträge über limnologische Probleme, 1891/92 berichtete Kantonsschulprofessor ARNET über physikalische Arbeiten und 1895 referierte BACHMANx über Planktonstudien am Vierwaldstätter- und Baldeggersee.

Das umfassende Programm für limnologische Untersuchungen, welches die von Forel begründete Commission d'études limnologiques herausgegeben hatte, wurde zum Vorbild des 1905 durch BACHMANN redigierten "Programms zur limnologischen Untersuchung des Vierwaldstättersees", unternommen von der Limnologischen Kommission der Schweizerischen Naturforschenden Gesellschaft, unter Mitwirkung einer entsprechenden Arbeitsgruppe der Naturforschenden Gesellschaft Luzern (NGL.).

Unter der initiativen Leitung des Basler Zoologen ZschokkE setzte eine rege Tätigkeit ein. Er und seine Schüler befassten sich mit zoologischen Untersuchungen. Die Luzerner ARNET und SCHLMACHER beschäftigten sich mit physikalischen und chemischen Fragen. BACHMANx setzte sich mit ungeheurem Eifer hinter das Studium des Phytoplanktons der Seen des Mittellandes und der Alpen. Daneben besuchte er 1905 Schottland, 1908 Grönland und 1914 Finnland, wo er reichlich Planktonproben sammelte. In der Zeit zwischen 1897 bis 1912 erschien von den oben Genannten eine groșse $Z$ ahl von Publikationen, die z.T. in den Mitteilungen der NGL zu finden sind. Wenn man alle diese Veröffentlichungen sorgfältig liest, fragt man sich: "Wann haben diese Luzerner Forscher, die alle einem Beruf verpflichtet waren, diese Arbeit geleistet, und wo und mit welchen Mitteln haben sie diese ungeheure Kleinarbeit vollbracht ?" Ein wohlausgerüstetes Laboratorium stand ihnen nicht zur Verfügung. Bereits 1910 entwarf Bachmann Pläne zur Errichtung eines Instituts für Gewässerfor- 
schung. Er dachte nicht an eine private oder kantonale Institution, ihm schwebte eine eidgenössische Forschungsstation vor. Am 31. Januar 1912 lancierte er eine Eingabe an das Eidg. Departement des Innern, betreffend das "Projekt für eine eidgenössische Station für Fischerei- und Gewässerkunde am Vierwaldstättersee». Beigelegt waren sorgfältig ausgearbeitete Pläne und eine wohldurchdachte Berechnung der Baukosten von Fr. 331250.- und der jährlichen Betriebskosten von Fr. 51000.-. Als Bauplatz war eine stille Bucht im Winkel bei Horw vorgesehen. Um die Bedeutung und Notwendigkeit eines solchen Instituts augenfällig zu machen, organisierte Bachmann 1911 und 1913 hydrobiologische Kurse, an denen Interessenten aus der Schweiz und den verschiedensten europäischen Ländern teilnahmen. Auch besuchte er im Ausland bereits bestehende limnologische Stationen, nahm Einblick in deren Einrichtungen und Betrieb und bekam dadurch die Grundlagen für die Aufstellung seiner Pläne. Das Departement des Innern versorgte die Eingabe in eine Schublade, 1914 brach der Erste Weltkrieg aus, und das Projekt blieb Wunschtraum.

Die Idee zu einem solchen Vorhaben wurde aber in Luzern weiterverfolgt, und man dachte zunächst an die Errichtung eines bescheidenen Laboratoriums durch die NGL. Rascher als man dachte, kam es zu einer Lösung. 1911 siedelte sich Dr. med. Fritz SCHWYZER, aus Amerika zurückgekehrt, in Kastanienbaum an. Bachmann fand in ihm einen aufgeschlossenen und für alle Probleme der Natur interessierten Freund. Er konnte Schwyzer für die Pläne zur Gründung einer limnologischen Station begeistern, und 1915 stellte dieser die Mittel für den Bau geschenkweise zur Verfügung. Am 18. Juni 1916 konnte das "Hydrobiologische Laboratorium» in Kastanienbaum feierlich eröffnet werden. Die NGL nahm es in ihre Obhut und setzte Bachmann als Kustos und wissenschaftlichen Leiter ein. Er versah diese Ämter ehrenhalber aber pflichtbewusst bis zu seinem Tode im Januar 1940. Zur Betreuung und Verwaltung wurde eine Hydrobiologische Kommission gewählt, die Bachmann als Präsident leitete. Die Besetzung der Stelle eines Assistenten bot Schwierigkeiten; entweder fehlte das Geld oder die geeignete Person.

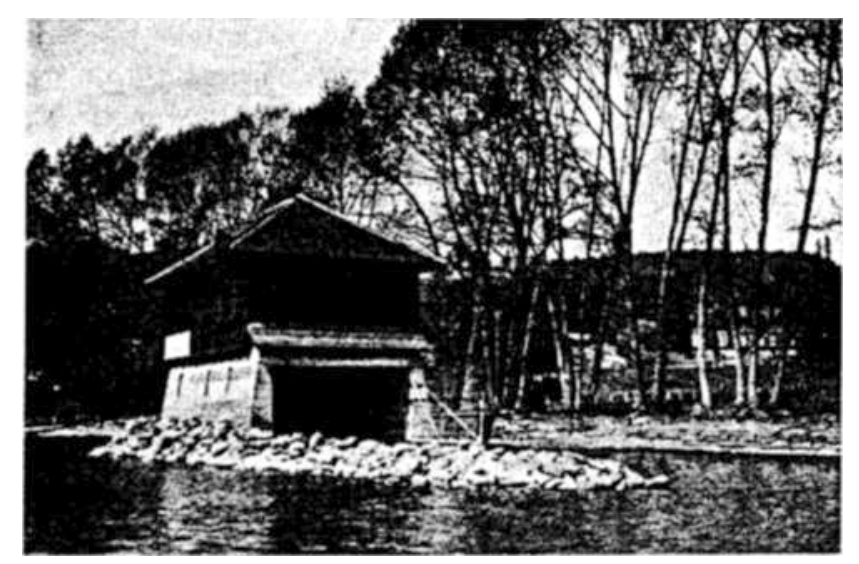

Abbildung 1

Das erste hydrobiologische Laboratorium in Kastanienbaum von 1916 bis 1938 . 
Die wissenschaftliche Arbeit Bachmanns möchte ich thematisch folgendermassen zusammenfassen:

1. Studien über technische und methodische Probleme. Anfänglich wurden Geräte verwendet, wie sie im Ausland für See- und Meeresforschung üblich waren. Sie erwiesen sich aber als ungenügend und vor allem als zuwenig präzis. In Zusammenarbeit Bachmanns und seiner Mitarbeiter mit der Firma Friedinger in Luzern ist von dieser eine grosse $\mathrm{Zahl}$ von Originalapparaten entwickelt worden (z. B. Planktonnetze, Theilersche Schöpfflasche, Kippthermometer, Planktonfilter u.a.m.), die sich als unentbehrliche Hilfsmittel erwiesen haben. Durch wissenschaftliche Publikationen und durch bebilderte Kataloge der Firma Friedinger wurden diese Apparate auch im Ausland bekannt. Die in Serie hergestellten Geräte wurden von der in allen Kontinenten einsetzenden hydrobiologischen Forschung angefordert.

2. Arbeiten über Morphologie, Systematik und Biologie des Phytoplanktons.

3. Vergleichende Phytoplanktonstudien der Seen Schottlands, Grönlands und der Alpen. Diese Studien veranlassten Bachmann zur Aufstellung der Seentypen der Schweiz.

4. Bearbeitung ausländischer Planktonproben, besonders solcher aus afrikanischen Gewässern.

5. Untersuchungen alpiner und hochalpiner Seen. Diese basieren auf zahlreichen selbstgesammelten Planktonproben aus zirka 60 Seen. Einen sehr breiten Raum nimmt die Erforschung des Ritom- und des Wägitalersees ein, deren Ergebnisse in der Schweizerischen Zeitschrift für Hydrologie, deren Redaktor Bachmann war, erschienen sind.

6. Probleme der Gewässerverschmutzung. Fortlaufende Planktonuntersuchungen um 1900 durch Hool liessen, vergleichend betrachtet, bereits 1910 eine zunehmende Veränderung des Planktonbestandes im Rotsee erkennen. Die Massenentwicklungen von Oscillatoria rubescens wurden immer auffälliger und dazu alle heute sattsam bekannten Begleiterscheinungen. Chemische Analysen ergaben eine erhebliche Zunahme des Nährstoffgehalts im Seewasser, und 1917 bezeichnete man diesen Zustand als Verschmutzung. Diese war verursacht durch die Einleitung grosser Mengen von Abwasser. Der Rotsee wurde Sorgenkind der Baudirektion der Stadt Luzern und für die folgenden Jahrzehnte ins Arbeitsprogramm des Laboratoriums aufgenommen. Bachmann, seine Mitarbeiter, das Kantonale Laboratorium und die Baudirektion schlossen sich zu einer Arbeitsgemeinschaft zusammen. Die Resultate der vorgenommenen Untersuchungen sind in einer grossen Zahl von Publikationen festgehalten, die in der Schweiz. Zeitschrift für Hydrologie und in den Berichten der NGL erschienen sind. Des weiteren hat sich Bachmann bis zum Jahre 1940 eingehend mit der Bekämpfung der Giftwirkungen von Mosterei- und Brennereiabwässern auf Fischgewässer befasst.

1924, anlässlich der 105. Jahresversammlung der SNG in Luzern, referierte Bachmann als Jahrespräsident über "Das Plankton der Schweizer Seen». Bei diesem Anlass würde er «in Würdigung seiner zahlreichen und vielseitigen Verdienste um die Förderung der Hydrobiologie» zum Ehrendoktor der Eidgenössischen Technischen Hochschule, Zürich, ernannt.

Das Laboratorium in Kastanienbaum bot aber nicht nur Bachmann ein Heim zu intensiver Forscherarbeit, sondern es diente auch vielen in- und ausländischen Spe- 
zialisten während Wochen oder Monaten als praktische Arbeitsstätte. Die Professoren SAUNders und Worthington aus Cambridge studierten den Einfluss der Wasserstoffionenkonzentration auf die Planktonverteilung und den Verlauf der Vertikalwanderungen des Planktons.

GJIMESI aus Budapest untersuchte mit Hilfe einer eigens dazu ausgedachten Filterpumpe das Nannoplankton, Jingar, Madras, und WAGELER, München, befassten sich mit Coregonenstudien. HURTER, Luzern, bearbeitete die Litoralalgen des Vierwaldstättersees und publizierte eine grössere Arbeit über Stechmückenbekämpfung. Weitere Arbeiten über den Vierwaldstättersee erschienen von BIRRER über die Rädertierchen und von SPÖRRI über die Infusorien des Litorals. Die drei eben Genannten waren abwechslungsweise als Assitenten am Laboratorium tätig. Gamma bearbeitete die makrophytische Uferflora und übergab sein wohlgeordnetes Herbarium in die Obhut des Laboratoriums. Surbeck, Bern, und Steinmann, Aarau, lieferten Beiträge zur Toxikologie der Fische. ZEMP, Luzern, untersuchte Planktonproben des Wägitaler-, Sarner- und Lungernsees. BIRRER befasste sich mit Studien am Weissfisch und Edelfisch. DüGGELI, Zürich, und ADAM, Luzern, benützten das Laboratorium zur Ausarbeitung eines Gutachtens über die Seewasserversorgung für die Gemeinde Horw. MUGGLIN und KNOPFLI, Luzern, oblagen ornithologischen Beobachtungen. JAAG, Zürich, studierte orientierungshalber die Planktonverhältnisse des Vierwaldstättersees. Die Konkordatskommission übertrug dem Laboratorium die Erstellung einer Fischereistatistik. Diese mühsame Arbeit übernahm ZEMP, der zusätzlich die zirka 30 Berufsfischer zweimal pro Jahr persönlich aufsuchen musste.

Zur Einführung in die Arbeitsweise und in die Probleme der Seeforschung führte SuRBECK mehrmals Exkursionen mit den Teilnehmern der Fischereilehrkurse durch. Portmann, Basel, und JAAG, Zürich, waren des öftern in Kastanienbaum Gäste, wo ihren Studenten die Durchführung von Planktonfängen, Messungen der Tiefentemperaturen und der Durchsichtigkeit des Seewassers u.a.m., praktisch gezeigt werden konnte.

Im Jahre 1936 berief Bachmann Seminarlehrer WoLfF, Luzern, als Assistenten an das Laboratorium, und vermittelte ihm, als Mitglied der Hydrobiologischen Kommission der Claraz-Schenkung, den Auftrag zur Untersuchung der Seen auf dem SanBernardino-Pass. Die umfangreichen Vorarbeiten zu dieser auf drei Jahre bemessenen Arbeit konnten in Kastanienbaum ausgeführt werden.

Bei der regen Benützung des kleinen Laboratoriums ergab sich bald ein empfindlicher Mangel an Arbeitsplätzen. Die Anhäufung der notwendigen Gerätschaften, die Vergrösserung der Bibliothek durch Fachschriften und aus aller Welt zugesandte Separata und dazu die ständige Zunahme der Sammlung von konservierten Planktonproben (seit 1930 wurden alle vierzehn Tage auf dem Kreuztrichter Proben entnommen), bedingten die Beschaffung zusätzlicher Räume, die in der Nähe gemietet werden konnten. Die Platzfrage war gelöst, ab̉er die konzentrierte Arbeit gestört. Dazu kam, dass die Besitzerin des Wieslandes zwischen Laboratorium und Strasse die Wegbenützung verweigerte. Im Laufe des Jahres 1937 stellten sich bei Bachmann ernsthafte Zirkulationsstörungen ein. Dazu kamen die eben beschriebenen unerfreulichen Zustände in Kastanienbaum. Dies bedrückte ihn sehr, und seine sonst stets initiative Schaffensfreude war gebrochen. 


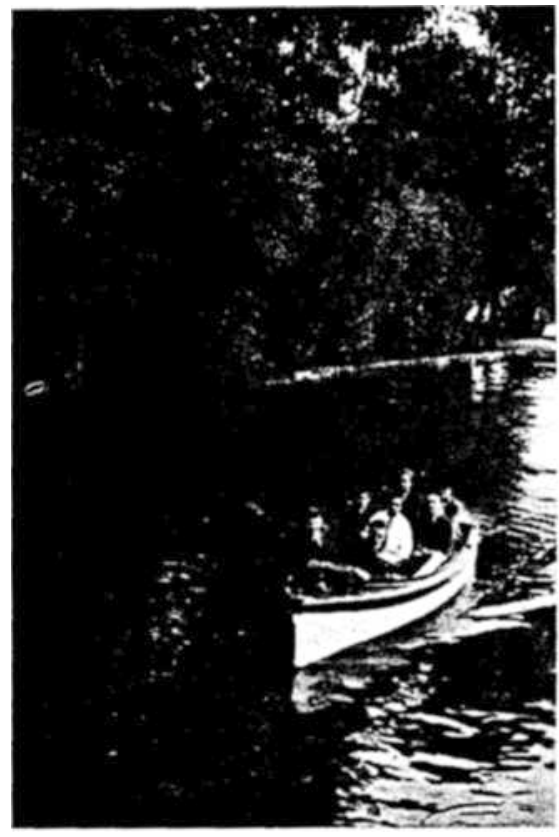

Abbildung 2

Ausfahrt mit dem Motorboot Charlotte anlässlich einer ETH-Exkursion im Juni 1937.

Im Frühjahr 1938 wurde durch die NGL eine Baukommission bestimmt und beauftragt, einen Bauplatz zu suchen, Pläne und finanzielle Mittel für einen Neubau zu beschaffen. Die Kommission arbeitete sehr speditiv, der Baubeschluss wurde gefasst, und die Bauarbeiten konnten unverzüglich aufgenommen werden. Anfangs September 1938 war der Bau beendet, und die feierliche Eröffnung des neuen Hydrobiologischen Laboratoriums fand bereits am 25. September statt. Im grossen, freundlichen Raum standen jetzt sechs Arbeitsplätze zur Verfügung. Alle Gerätschaften, die Bibliothek und die Probensammlung fanden in Schränken und Regalen ihren Platz. $\mathrm{Zu}$ Beginn des folgenden Jahres hatte sich Bachmann von seinen Beschwerden gut erholt, und der Schreibende schloss Ende Sommer seine Arbeit auf dem San-Bernardino-Pass ab. Wir begannen mit der Untersuchung der reichlich gesammelten Planktonproben. Aber im Herbst 1939 brach der Zweite Weltkrieg aus, und mit der Arbeit war es vorbei. Alle Mitarbeiter rückten zum Aktivdienst ein, und Bachmann blieb allein zurück. In der Folge wurde der Schiffsverkehr auf dem Vierwaldstättersee so stark eingeschränkt, dass das Laboratorium kaum noch zu erreichen war, und das war für ihn eine schwerwiegende Enttäuschung.

Am Abend des 20. Februar 1940, mitten im Gespräch mit Bekannten und ohne vorherige Anzeichen, starb Prof. Hans Bachmann. In einer Gedenkfeier am 9. November 1940, im Rahmen der Naturforschenden Gesellschaft Luzern, wurden seine Verdienste als Lehrer, Hydrobiologe und langjähriger Präsident der NGL eingehend gewürdigt. 


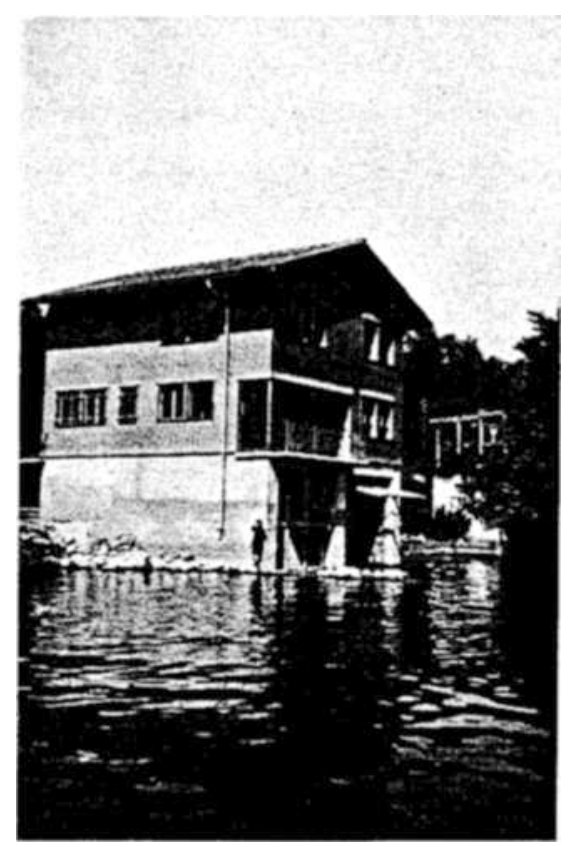

Abbildung 3

Das neue hydrobiologische Laboratorium vom See her gesehen. September 1938.

Im Frühjahr 1940 übernahm Kantonschemiker Dr. FRITZ AdAM das Präsidium der Hydrobiologischen Kommission, und Seminarlehrer Dr. Heinrich WolfF wurde zum Kustos und wissenschaftlichen Leiter des Laboratoriums ernannt. Wir versuchten nun zwischen Aktivdienst und Beruf das Institut so gut als möglich zu betreuen und die Arbeit im Sinn und Geist Bachmanns fortzuführen. Zunächst erfolgte eine Umordnung der Bibliothek nach Fachgebieten und die Herstellung einer nach Autoren geordneten Kartothek. WoLfF studierte die Entwicklungszyklen und die Art der Überwinterung bei Cladoceren, Copepoden und Rotatorien der San-Bernardino-Seen. BurckHaRdT, Basel, führte uns während einer Woche in die Bestimmungsmethoden für das Zooplankton ein. Er war damals der beste Kenner dieser Kleintierformen schweizerischer Seen. ZEMP übernahm wieder das Amt eines Assistenten. Er führte an 400 Fischen aus dem Zugersee Alters- und Geschlechtsbestimmungen durch und stellte eine Liste über die Molluskenarten aus der Seekreide von Wauwil auf. StEInmans, Aarau, benützte das Laboratorium zur Untersuchung von Frühreifeerscheinungen an Weissfischen. GROB, Zürich, fasste die Bestimmungsergebnisse für das Plankton des Pfäffikersees in einer Diplomarbeit zusammen.

$\mathrm{Zu}$ Beginn des Jahres 1942 wurde es zusehends ruhiger in Kastanienbaum. Schuld daran trugen die immer wiederkehrenden Aktivdienste und das seltener werdende Interesse für hydrobiologische Forschungen bei Botanikern und Zoologen unserer Universitäten. Wir dachten an die Durchführung von Studentenkursen und setzten uns mit Prof. Portmans in Basel in Verbindung. Er sagte augenblicklich zu, und unter 


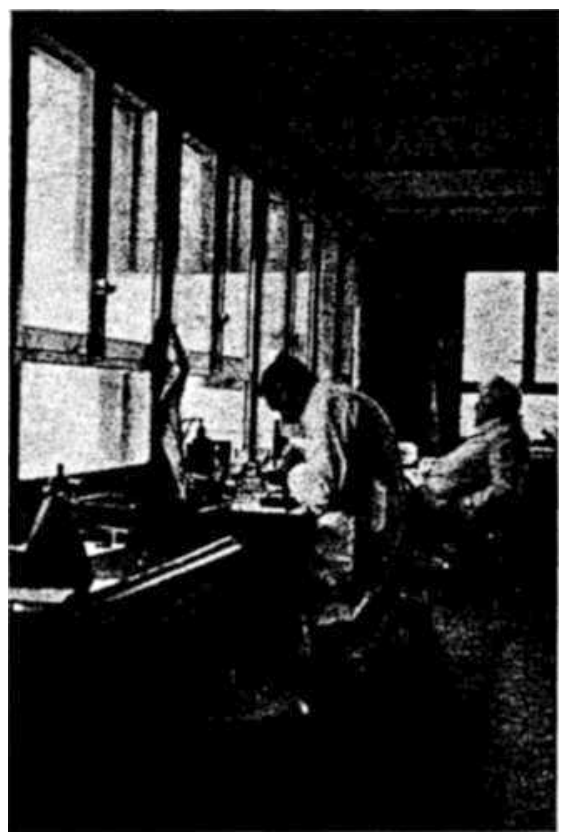

Abbildung 4

Blick in den grossen Arbeitsraum. Im Hintergrund Prof. Bachmann. November 1938.

der gemeinsamen Leitung von Portmann und Wolff konnte vom 1. bis 10. September 1942 der erste Ferienkurs mit 16 Studierenden von Basel, Bern und Zürich abgehalten werden. Im April 1944 folgte ein zweiter Kurs, an dem 25 Studierende teilnahmen. 1946 nahmen 15 Sekundarlehrer aus dem Kt. Luzern unsere Einladung zu einer Kurswoche an, und 1947 waren 18 Gymnasiallehrer in Kastanienbaum beisammen. Zur Bereicherung des Kursprogramms verpflichteten wir Fachleute, die die Vielschichtigkeit der hydrobiologischen Forschung darlegten. Besonders attraktiv waren jeweils die durch Fischereiaufseher HoFER, Meggen, vorgeführten Fischfangmethoden. Durch die Bereitstellung finanzieller Mittel bekundeten Kanton und Stadt Luzern ihr Interesse für diese Kurse.

Mitte der vierziger Jahre gelang es uns, Sekundarlehrer R. VOLLENwEIDER für die Hydrobiologie zu begeistern und als Assistenten zu gewinnen. Angeregt durch die Kurse und durch intensives Studium der neueren Literatur beschlossen wir, der wissenschaftlichen Arbeit neuen Auftrieb zu geben. Durch die Besuche verschiedener wissenschaftlicher Gesellschaften in Kastanienbaum kamen wir mit Fachgelehrten ins Gespräch, wobei wir manche wertvolle Anregungen zum Studium der Gewässerprobleme entgegennehmen durften. 1945 hielt die Schweiz. Botanische Gesellschaft in Luzern und Kastanienbaum ihre Frühjahrsversammlung ab. 1947 besuchte uns die Naturforschende Gesellschaft Solothurn. 1948 konnten wir die Mitglieder des Kongresses der Internationalen Limnologen-Vereinigung empfangen und wertvolle Verbindungen mit ausländischen Kapazitäten aufnehmen. 1949 beehrte uns Prof. STEINER, 


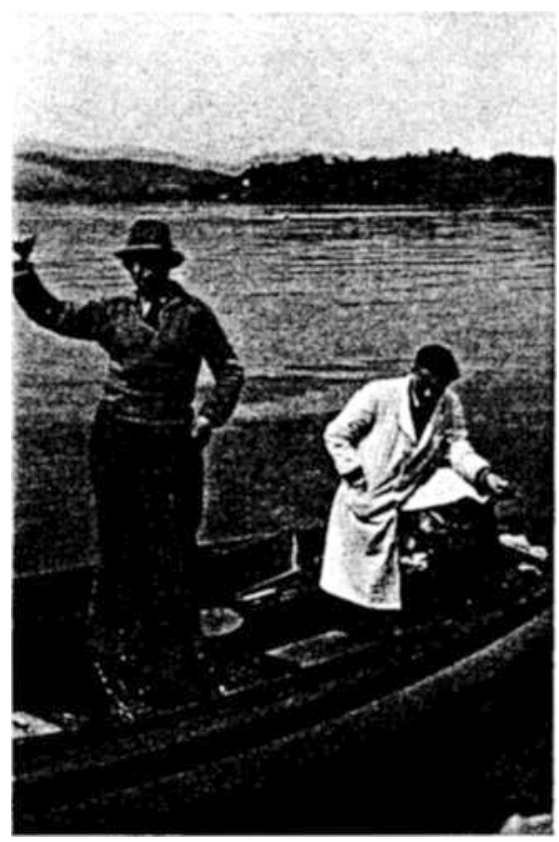

Abbildung 5

Der wissenschaftliche Leiter Dr. H. Wolff und Assistent R. Vollenweider bei einer Gerätedemonstration auf dem Kreuztrichter, anlässlich einer Exkursion der Naturforschenden Gesellschaft Luzern.

Zürich, mit einer Zoologenexkursion. 1950 nahm die Gesellschaft der Fischermeister Einblicke in die Arbeit der Hydrobiologen. 1951, wie schon in früheren Jahren, besuchte uns Prof. JAAG mit seinen Studenten. Im selben Jahr hielt die Schweiz. Naturforschende Gesellschaft ihre Jahresversammlung in Luzern ab. Wir organisierten eine Exkursion nach Kastanienbaum, wobei etwa 50 Teilnehmer Einrichtungen und Arbeitsweise unseres Institutes kennen lernen konnten.

Nach 1945 war es möglich, auch die wissenschaftliche Tätigkeit wieder zu intensivieren. Zunächst wurden die auf San Bernardino gesammelten Proben und Daten verarbeitet. Dann beschäftigten uns die seit Jahren beobachteten Veränderungen im Planktonbestand unserer Seen, gekennzeichnet durch das Verschwinden einzelner Algenarten und die immer häufiger auftretenden Massenentwicklungen von Oscillatoria und einiger Diatomeenarten. Die Ursache dieser Erscheinungen war offensichtlich die zunehmende Verschmutzung unserer Gewässer. Um nun die Einwirkung der ständig zunehmenden mineralischen und organischęn Substanzen auf die Entwicklung einiger Algenarten studieren zu können, hatten wir uns entschlossen, mit Hilfe von Reinkulturen zu experimentieren. Die dabei angewandte Methodik und die erhaltenen Resultate hat VOLLENWEIDER in seiner Dissertation zusammengefasst. Die völlige Umstellung der Arbeitsweise verlangte eine Neugestaltung verschiedener Räume. Die finanziellen Mittel zu diesem Umbau wurden von der Direktion der Centralschweizerischen Kraftwerke sowie vom Leiter des Laboratoriums zur Verfügung gestellt. 
Zwischen 1945 und 1950 sind folgende Publikationen in der Schweiz. Zeitschrift für Hydrologie erschienen:

WolfF, HсH., Hydrobiologische Untersuchungen an den hochalpinen Seen des SanBernardino-Passes.

VOLLENWEIDER, R., Zum Gesellschaftsproblem in der Limnobiocoenologie, überdies

Neue Gesichtspunkte zur Durchführung von Planktonuntersuchungen und öologische Untersuchungen von planktischen Algen auf experimenteller Grundlage.

VollenweIder und WolfF, Zur Methodik der Planktonstatistik und ein Schlagwortverzeichnis der Bde. $I-X$ der Schweiz. Zeitschrift für Hydrologie.

In den verflossenen zehn Jahren hatte sich der wissenschaftliche Leiter mit verschiedenen kleineren und grösseren Untersuchungen zu befassen, z. B. am Rotsee, an dessen Auslauf, dem Ronbach, über die Ursache und Sanierung einer durch Eisenbakterien verschmutzten Quellfassung bei der Wasserversorgung in Winterthur, über die Herkunft des Wassers bei Stolleneinbrüchen in Garegna und am Ritomsee u.a.m. Solche Aufträge kamen meist überraschend und hatten oft tagelange Aufenthalte an Ort und Stelle zur Folge. Die Resultate dieser Untersuchungen wurden in Form von Gutachten zusammengefasst. Die durch solche Aufträge erhaltenen Honorare flossen in die Institutskasse. Öfters wurde der Leiter von Körperschaften und Vereinen der verschiedensten Interessengruppen eingeladen, Vorträge zu halten. Hierbei bot sich die Gelegenheit, auf die fortschreitende Verschmutzung unserer öffentlichen Gewässer hinzuweisen und den dringend notwendigen Einsatz der wissenschaftlichen Forschung zu fordern.

Die sprunghafte Entwicklung der Gewässerforschung, die sich anfangs der fünfziger Jahre in verschiedenen Ländern Europas aufzeigte, stellte uns vor neue entscheidende Probleme. Wir mussten einsehen, dass eine mit dem Ausland konkurrenzfähige Forschung einen weiteren Ausbau des Laboratoriums und die Anschaffung moderner Gerätschaften erforderte. Ferner musste die Anstellung eines vollamtlich bezahlten

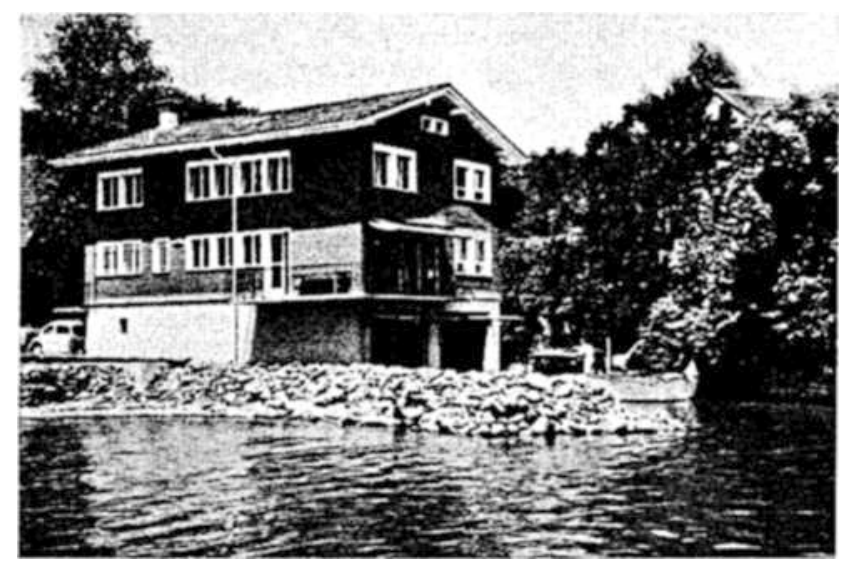

Abbildung 6

Das umgebaute und vergrösserte hydrobiologische Laboratorium Kastanienbaum im Jahre 1964. (Foto: EAWAG) 
und qualifizierten Wissenschaftlers vorgesehen werden. Bis jetzt arbeiteten alle Mitarbeiter einschliesslich Leiter ehrenamtlich, nur die Stelle des Assistenten war bescheiden honoriert. Die Mittel, über die die NGL zum Betrieb des Laboratoriums verfügte, flossen aus spärlichen Subventionen von Kanton und Stadt Luzern, aus Zuwendungen der Stiftung für Suchende, der Centralschweizerischen Kraftwerke und weiterer Industriebetriebe. Die letzte durchgeführte Bettelaktion ergab Fr. 5765.-, dazu kamen noch in Aussicht gestellte Subventionen von zirka Fr. 1500.-. Der auf neuer Basis errechnete Kostenvoranschlag ergab eine Anlaufsumme von Fr. 50000.-, ein Betrag, den die NGL nicht aufbringen konnte. Damit war die verantwortbare Weiterführung des Instituts in Frage gestellt. Die oben beschriebene Sachlage und die zunehmende Beanspruchung im Lehrberuf veranlassten den wissenschaftlichen Leiter, 1953 von seinem Amte zurückzutreten. Dieses Amt wurde in der Folge nicht mehr besetzt. VOLLENWEIDER arbeitete noch ein Jahr unter Mitwirkung einiger Schulkollegen weiter. 1954 verliess auch er Kastanienbaum und verpflichtete sich für einige Jahre an das Limnologische Institut in Pallanza.

Die letzten Publikationen aus dieser Zeit:

VollenWeIDER, R., Aspekte der modernen Limnologie (erschienen in Mitteilungen der NGL 1951),

Einige Bemerkungen zur ökologischen Valenzanalyse (erschienen in Schweiz. Z. Hydrol. 1953).

VOLLENWEIDER, R. und FreY, M., Verteilung der Leitfähigkeit in einem eutrophen See während der Sommerstagnation.

WoLF, K., Morphometrische Studien an Daphnia longispina O. F. Müller und Quantitative Untersuchungen am Zooplankton des Rotsees (beide Artikel erschienen in Schweiz. Z. Hydrol. 1955).

Die Hydrobiologische Kommission der NGL versuchte in der Folge, durch Anstellung von Studierenden als Assistenten den Betrieb im Laboratorium aufrechtzuerhalten. Diese jungen Leute erhielten aber keine Anleitung zur Arbeit, blieben unbeaufsichtigt, und es ergaben sich dadurch viele Ungelegenheiten. 1956 befasste sich der Vorstand der NGL mit der Sachlage und erkannte die Aussichtslosigkeit der Weiterführung des Laboratoriums im Rahmen der Gesellschaft. Auch waren die bisherigen Donatoren nicht in der Lage, sich auf die Bereitstellung grösserer Mittel auf Jahre hinaus zu verpflichten. Man erinnerte sich an die Idee Bachmanns, eine eidgenössische Station für Gewässerforschung zu errichten, und beschloss, mit der Direktion der Eidg. Anstalt für Wasserversorgung, Abwasserreinigung und Gewässerschutz an der Eidg. Technischen Hochschule in Zürich Verbindung aufzunehmen. Im Sommer 1957 fanden die ersten Besprechungen mit Prof. JAAG und dem Präsidenten des Schweizerischen Schulrates, Prof. Pallmann, statt. In der Folge entschloss sich der Vorstand der NGL, das Laboratorium unter bestimmten Vorbehalten der ETH als Geschenk zu offerieren. Prof. Jaag übernahm dann das Institut vom Juni 1958 bis Frühjahr. 1960 versuchsweise. Im März 1960 hatte der Bundesrat die Offerte entgegengenommen und die Übernahme bewilligt, und im Sommer des gleichen Jahres wurde die Urkunde unterzeichnet; die Übergabe war perfekt. Der Traum Bachmanns war in Erfüllung gegangen. Aber eines müssen wir Luzerner hier noch beifügen: wir trauern sehr um unser Hydrobiologisches Laboratorium, das während 44 Jahren im Mittelpunkt des wissenschaftlichen Geschehens unserer Gesellschaft stand. Aber wir freuen 
uns heute aufrichtig, dass dieses Institut unter würdige Obhut gekommen und sein Weiterbestehen gesichert ist. Wir danken Herrn Prof. Jaag für alle seine Bemühungen und wünschen dem Hydrobiologischen Laboratorium der ETH in Kastanienbaum eine erfolgreiche Zukunft.

Die hier wiedergegebene Geschichte des Hydrobiologischen Laboratoriums Kastanienbaum wurde, auf Veranlassung von Prof. Dr. O. Jaag, als Referat den schweizerischen Mitgliedern der Internationalen Vereinigung für Limnologie am 3. Oktober 1964 in Kastanienbaum vorgetragen. 